\title{
La actividad de aprendizaje de matemáticas en ingeniería de alimentos: constitución del sentido personal del estudiante ${ }^{1}$
}

\author{
The math learning activity in food engineering: \\ constitution of the student's personal sense
}

\begin{abstract}
A matemática atividade em engenharia de alimentos de aprendizagem: criação de sentido pessoal de estudante
\end{abstract}

Recibido: mayo de 2013

Aceptado: agosto de 2013

\section{Resumen}

Este escrito es un proyecto de investigación de Maestría en Educación Matemática. El objetivo que orienta dicha investigación es analizar el proceso de constitución del sentido personal de los estudiantes de ingeniería de alimentos desde y hacia la actividad de aprendizaje de matemáticas. La aproximación teórico-metodológica se fundamenta en las contribuciones de la perspectiva histórico-cultural de la educación y de la Teoría de la Actividad. Dicha aproximación se basa una metodología enmarcada en una investigación colaborativa, el grupo colaborativo está conformado por el investigador, el profesor del curso y algunos estudiantes. Los registros y datos se producen por los estudiantes y el investigador a partir de diarios de campo, autobiografías, análisis de episodios, ideogramas, textos escritos, mapas conceptuales y entrevistas.

Palabras clave: Educación Matemática y otras disciplinas; Aprendizaje; Ingeniería; Sentido personal; Perspectiva Histórico-cultura de la educación; investigación e innovación en matemáticas; marcos teóricos; teorías de aprendizaje; situado sociocultural.

\begin{abstract}
This paper is a research Masters in Mathematics Education. The goal that guides this research is to analyze the process of formation of the students' personal sense of food engineering and from the learning of mathematics. The theoretical-methodological approach is based on the contributions of historical and cultural perspective of education and Activity Theory. Such a methodology is based approach framed in collaborative research, collaborative group consists of the researcher, the course teacher and some students. The records and data produced by the students and the researcher from field diaries, autobiographies, analysis of episodes, ideograms, written text, concept maps and interviews.
\end{abstract}

1 Artículo de Investigación. Este trabajo es orientado de forma compartida por las profesoras: Dra. Diana Victoria Jaramillo Quiceno (djaramillo.quiceno@hotmail.com) y Dra. Lucia Zapata Cardona (minervaluka@hotmail.com), Facultad de Educación, Universidad de Antioquia.

2 Universidad de Antioquia. Medellin, Colombia. Estudiante de Maestría en Educación. Contacto: rigo.villada@gmail.com. 
Keywords: ICT, Mathematics Education Other concepts, problem solving, resolution of problem situations; them de recherche narrations, mathematical language; classroom teaching resources, computing resources, Internet, communication platform.

\section{Resumo}

Este trabalho é uma pesquisa de Mestrado em Educação Matemática. O objetivo que norteia esta pesquisa é analisar o processo de formação de sentido pessoal dos alunos de engenharia de alimentos e da aprendizagem da matemática. A abordagem teórico-metodológica baseia-se nas contribuições da perspectiva histórica e cultural do ensino e da Teoria da Atividade. Tal metodologia é a abordagem enquadrado na pesquisa colaborativa baseada, grupo colaborativo consiste em o pesquisador, o professor do curso e alguns alunos. Os registros e dados produzidos pelos alunos ea pesquisadora de diários de campo, autobiografias, análise de episódios, ideogramas, texto escrito, mapas conceituais e entrevistas.

Palavras-chave: Educação Matemática e outras disciplinas, a aprendizagem, Engenharia, sentido pessoal, cultura Perspectiva histórica da educação, investigação e inovação em matemática, referenciais teóricos, as teorias de aprendizagem, situado sociocultural.

\section{Planteamiento del problema}

La ingeniería de alimentos tiene un objeto de estudio propio pero considero que los ingenieros que ejercen como profesores de este campo deben inquietarse por cuestiones relacionadas con la enseñanza y el aprendizaje. Esto no significa abandonar la especificidad del objeto de estudio de este campo de la ingeniería sino asumir otras problemáticas que son inherentes a su labor como profesores en ingeniería. Esas problemáticas demandan que los profesores asuman una posición teórica y epistemológica que les posibilite mirar y comprender el aprendizaje de sus estudiantes. Es así como esta propuesta quiere ofrecer una mirada de cómo acontece el aprendizaje de las matemáticas en los estudiantes de ingeniería de alimentos.

Este proyecto de investigación propone una mirada relacionada con el "sentido personal" atribuido por el estudiante de ingeniería de alimentos al aprendizaje de las matemáticas. Al revisar otras propuestas encuentro que algunos investigadores en la educación de ingenieros (Christensen, 2008; Duque, 2008; Graaff, 2008 ; Wankat \& Oreovicz,

1993) perciben igualmente que el aprendizaje es un problema de "sentido". Sin embargo, la perspectiva desde la cual es visto este "sentido" lo relaciona con la capacidad que tiene el estudiante para comprender la información que recibe por sus sentidos fisiológicos y que finalmente es procesada por el cerebro. En definitiva, considero que la perspectiva de estos autores, aunque se preocupa por el aprendizaje del estudiante, ve al estudiante en una sola dimensión, por lo tanto estoy de acuerdo con Valero (2002) cuando sugiere:

\begin{abstract}
Nuestros estudiantes no son solamente "cabezas"-léase sujetos cognitivos- sino que son seres con una existencia física y temporal, con sentimientos, con múltiples razones para involucrarse (o no) en el aprendizaje de las matemáticas, y con una vida que trasciende los límites del aula. (p.55)
\end{abstract}

Así, en esta propuesta, asumo el aprendizaje de matemáticas desde los principios teórico-metodológicos de la Teoría de la Actividad, en la cual el aprendizaje es visto como un proceso que sucede en la medida que el estudiante le atribuye 
un "sentido personal" a su que-hacer académico. De acuerdo con Asbahr (2011a), el concepto de "sentido personal" amplía la comprensión sobre el aprendizaje y posibilita comprender al estudiante como un sujeto que piensa, actúa, siente y escoge de acuerdo al sentido personal que le atribuye a sus conocimentos.

Bajo esta perspectiva de Asbahr (2011a), en esta propuesta de investigación me aproximaré al proceso de constitución del sentido personal en el estudiante desde y hacia la actividad de aprendizaje de matemáticas en ingeniería de alimentos. El sentido personal hacia la actividad de aprendizaje, lo comprendo, como la significación personal que tiene esta actividad para el estudiante, una significación que se va creando constantemente y que está vinculada a su experiencia de vida. (Asbahr, 2011a, 2011b; Rigon, Asbahr \& Moretti, 2010).

Teniendo en cuenta lo anterior, y al observar las dinámicas de la clase tradicional en ingeniería de alimentos, encuentro que los estudiantes efectivamente realizan acciones en búsqueda de un objetivo de aprendizaje. Sin embargo, estas acciones están orientadas por motivos ajenos a la apropiación del conocimiento, como por ejemplo: hacer lo que indica el profesor, aprobar u obtener una buena nota definitiva en el curso. Por lo tanto, está situación me lleva a cuestionarme sobre como las clase de matemáticas se constituye en una experiencia de vida para los estudiantes, que les posibilite crear sentidos personales hacia el aprendizaje de las matemáticas relacionados con la apropiación del conocimiento matemático.

De esta manera, la pregunta de investigación que he configurado para el proyecto es: ¿Cómo se constituye el sentido personal de los estudiantes desde y hacia la actividad de aprendizaje de matemáticas en ingeniería de alimentos?

\section{Marco Teórico}

La Teoría de la Actividad se sustenta en los principios filosóficos del materialismo histórico-dialéctico. El concepto de actividad explica teóricamente toda la práctica humana universal, y posibilta entender el desarrollo histórico de la cultura (Rigon et al., 2010; Davidov, 1988). Para Davidov (1988), la esencia del concepto de actividad, es la relación del sujeto como ser histórico y social hacia la realidad externa. Esta relación se concretiza en el trabajo, que es aquello que genera la transformación de dicha realidad a través de la creación de instrumentos dirigidos a una finalidad, la humanización del sujeto.

Con base en la Teoría de la Actividad, la actividad humana se convierte en el foco de análisis para la comprensión de los procesos de desarrollo humano presentes en la educación, que a la vez está constituida por un complejo sistema de actividades (actividad de aprendizaje y actividad de enseñanza) (Moura, Araújo, Ribeiro, Panossian \& Moretti, 2010).

Según Asbahr (2011a), la apropiación por los estudiantes de los conocimientos se torna como objeto y motivo de la actividad de aprendizaje del estudiante. Por lo tanto, para que el objeto pueda ser concretizado por el sujeto, el objeto debe ser el motivo de la actividad y debe estar relacionado con el objetivo de las acciones emprendidas por el sujeto. Así es como el sentido personal hacia la actividad se crea desde la actividad, ya que, el sentido personal es "la relación objetiva entre lo que incita a la acción en el sujeto y aquello para lo cual su acción se orienta, traduciendo la relación entre el motivo y el fin de la actividad" (Asbahr, 2011a, p.1).

El sentido personal hacia la actividad de aprendizaje se constituye cuando dicha actividad ocupa un lugar en la vida real del sujeto y no es solamente una respuesta a condiciones externas, impuestas por otras personas o situaciones. Cuando la actividad no tiene un sentido real, conectado a los motivos del propio estudiante, la actividad se torna formal, meramente reproductiva (Asbahr, 2011a, 2011b).

\section{Metodología}

Busco aproximarme al proceso de constitución del sentido personal enfocando mi mirada sobre 
la actividad de aprendizaje del estudiante de ingeniería de alimentos, bajo una propuesta metodológica de carácter cualitativo. Con relación a los principios teórico-metodológicos de la perspectiva histórico-cultural de la educación y la Teoría de la Actividad, resalto lo que manifiestan Rigon et al. (2010):

En el caso de la investigación en educación cuyo objeto es la actividad pedagógica manifestada en las relaciones entre enseñanza y aprendizaje, se requiere investigar las acciones de profesores y estudiantes no solo de manera descriptiva, sino fundamentalmente comprendiendo el origen de esas acciones, los motivos de la actividad y cuales son los sentidos atribuidos. (p.41)

Para poder responder a la pregunta planteada en esta propuesta considero pertinente desarrollar una investigación colaborativa. Así, propongo la conformación de un grupo colaborativo en el curso de Balances de Masa y Energía entre el investigador, el profesor y los estudiantes. De acuerdo con Boavida \& Ponte (2011), la colaboración es entendida como trabajo conjunto, de modo que los objetivos se logren a partir de la ayuda mutua pero en una relación no jerárquica ni utilitarista. Además, la investigación colaborativa tiene varias ventajas que radican principalmente en que la diversidad de perspectivas y experiencias de los integrantes del grupo aportan unas condiciones que posibilitan, a partir de la reflexión y el diálogo, el conocimiento mutuo, la generación de propuestas innovadoras de cambio y la superación de inconvenientes durante la aplicación de estas propuestas.

Los registros y datos se producen por los estudiantes y el investigador a partir de instrumentos como diarios de campo, autobiografías, análisis de episodios o casos, ideogramas, textos escritos, mapas conceptuales y entrevistas. El análisis de los datos se realizará a partir de los enunciados escritos y orales producidos por los estudiantes durante los encuentros del grupo colaborativo, en triangulación con la teoría y la perspectiva, en constitución, del investigador.

\section{Referencias}

Asbahr, F. (2011a). Por que aprender isso professora? Sentido pessoal e atividade de estudo na Psicologia Histórico-Cultural. Tesis doctoral, Universidade de São Paulo, São Paulo.

Asbahr, F. (2011b). Sentido pessoal, significado social e atividade de estudo: Una revisão teórica. Recuperado el 14 de Octubre de 2012, de V Ebem Encuentro Brasileiro de Educação e Marxismo : http://www.5ebem. ufsc.br/trabalhos/eixo_04/e04c_t001.pdf

Boavida, A. \& Ponte, J. (2011). Investigación colaborativa: Potencialidades y problemas. Revista Educación y Pedagogía, 23(59), 125-135.

Cedro, W. \& Moura, M.O. (2004). O espaço de aprendizagem e a atividade de ensino: o clube de matemática. Recuperado el 10 de Octubre de 2012, de Sociedade Brasileira de Educação Matemática (SBEM): http://www.sbem. com.br/files/viii/pdf/02/CC78728770153. pdf

Christensen, H. (2008). De la actividad al aprendizaje activo. En ACOFI, Ciencia e Ingeniería en la Formación de Ingenieros para el siglo XXI (p. 87-116). Bogotá: ACOFI.

Davidov, V. (1988). La enseñanza escolar y el desarrollo psíquico. Moscú: Editorial Progreso.

Duque, M. (2008). Principios para la enseñanza compatibles con el aprendizaje. En ACOFI, Ciencia e Ingeniería en la formación de ingenieros para el siglo XXI (p. 17-41). Bogotá: ACOFI.

Graaff, E. (2008). Aprendizaje basado en problemas en ingeniería. En ACOFI, Ciencia e Ingeniería en la Formación de Ingenieros para el siglo XXI (p. 117-127). Bogotá: ACOFI. 
Moura, M.O.; Araújo, E.; Ribeiro, F.; Panossian, F. \& Moretti, V. D. (2010). A Atividade Orientadora de Ensino como unidade entre ensino e aprendizagem. En M. Moura, A Atividade Pedagógica na Teoria HistóricoCultural (págs. 81-109). Brasília DF: Liber livro.

Rigon, A. J.; Asbahr, F. \& Moretti, V. D. (2010). Sobre o procceso de humanização. En M. O.
Moura, A Atividade Pedagógica na Teoria Histórico-Cultural (págs. 7-35). Brasilia: Liber Livro Editora Ltda.

Valero, P. (2002). Consideraciones sobre el contexto y la educación matemática para la democracia. Quadrante, 11(1), 49-59.

Wankat, P. \& Oreovicz, F. (1993). Teaching Engineering. Purdue University. 